\title{
The efficacy of slide tracheoplasty in a growing rabbit model
}

\author{
Yoichi Takano $\cdot$ Kosaku Maeda $\cdot$ Chieko Hisamatsu • \\ Eiji Nishijima
}

Published online: 19 October 2011

(c) The Author(s) 2011. This article is published with open access at Springerlink.com

\begin{abstract}
Purpose Slide tracheoplasty for congenital tracheal stenosis was reported by Tsang. This procedure provides good results, but there are few studies about the tracheal growth of post-surgical condition. Recently, not only traditional posterior to anterior slide plasty but also right side to left side slide plasty was reported. We evaluated the tracheal growth after slide tracheoplasty in growing Japanese white rabbit model.

Methods The Japanese white rabbits, weighing 1,600$2,400 \mathrm{~g}$, were used for this study. One-third of the estimated length of the trachea was slantingly resected about an appropriate angle. We performed a slant, side-to-side tracheal anastomosis in two ways (slanted from the front to the back in four animals, and slanted from right to left in four animals) on growing rabbits as an animal model of slide tracheoplasty.

Results All the rabbits were alive until the 10th week after surgery. There was no evidence of differential growth in any part of the circumference of the normal and anastomotic tracheas. Histologically, all tracheal lumina were completely lined with normal respiratory epithelium and normal surrounding cartilage.
\end{abstract}

Y. Takano $\cdot$ C. Hisamatsu $\cdot$ E. Nishijima

Department of Pediatric Surgery, Kobe University Graduate

School of Medicine, 7-5-2 Kusunoki-cho, Chuo-ku,

Kobe 650-0017, Japan

Y. Takano $\cdot$ K. Maeda $(\bowtie)$

Division of Pediatric Surgery, Jichi Medical University School

of Medicine, 3311-1 Yakushiji, Shimotsuke,

Tochigi 329-0498, Japan

e-mail: maedak@jichi.ac.jp
Conclusion The results presented that these two methods did not prevent the tracheal growth and trachea did not buckle macroscopically. Both slide tracheoplasties did not interrupt the growth of trachea.

Keywords Congenital trachea stenosis - Slide tracheoplasty $\cdot$ Tracheal growth $\cdot$ Animal model

\section{Introduction}

Congenital tracheal stenosis is a life-threatening disease. Several types of surgery have been introduced for this disease, such as balloon dilatation, segmental resection, and patch tracheoplasty with pericardium and costal cartilage $[1,2]$. However, for the long-type tracheal stenosis, these various types of surgery do not provide sufficient results.

Slide tracheoplasty for congenital tracheal stenosis was first reported by Tsang [1]. This procedure provides good results, but there have been few studies about the tracheal growth during the post-surgical condition [3, 4]. Recently, not only traditional posterior to anterior slide plasty but also right side to left side slide plasty was reported, thus indicating that the procedure will more likely be used more in future.

We herein evaluated the tracheal growth after slide tracheoplasty in growing a Japanese white rabbit model.

\section{Materials and methods}

Special humane animal care measures were taken for the rabbits in compliance with the "Guide for animal experimentation" (Jichi Medical University) and the "Guide for 
the care and use of laboratory animals" (National Institute of Health, 1985). The number of animals was limited to the minimum number necessary to obtain useful results.

Japanese white rabbits weighing $1,600-2,400 \mathrm{~g}$ were used for this study. The animals were anesthetized with intravenous administration of midazolam $(1.5 \mathrm{mg} / \mathrm{kg})$ and inhaled diethyl ether. They were placed in the supine position under spontaneous ventilation without an endotracheal tube. The entire trachea which measured $3 \mathrm{~cm}$ in length was then exposed through a midline cervical incision, and one-third of the estimated length of the trachea was slantingly resected on an appropriate angle. A $1-\mathrm{cm}$ segment of the trachea is comprised about one-third of the entire length of the trachea in this animal model. We then performed a slant side-to-side tracheal anastomosis in two ways, using the growing rabbits as an animal model of slide tracheoplasty. We consider that this animal model closely mimics human slide plasty in long congenital tracheal stenosis. The diagonal pair of tracheas was sutured using interrupted absorbable monofilaments (5-0 PDS II ${ }^{\circledR}$ ) and the wounds were closed in the usual fashion (Figs. 1, 2). After surgery, the animals did not require any special care, such as sedation or respiratory management.

The animals were classified into three groups: in group A, the trachea was resected by scissors, and slanted from the front to the back $(n=4)$, in group B, the trachea was resected by scissors, then slanted from right to left $(n=4)$, and in group C, no surgery was performed, and the animals served as controls $(n=3)$.

All specimens were removed 10-11 weeks after surgery. The external diameter of the specimens was measured with calipers, and we performed bronchoscopy at the time of harvesting. A pathologic examination was also performed with hematoxylin and eosin ( $\mathrm{H} \& \mathrm{E})$ staining, and Elastica van Gieson staining.

Fig. 1 The surgical procedure for the animal model of slide tracheoplasty (group A: front to back, group B: right to left)

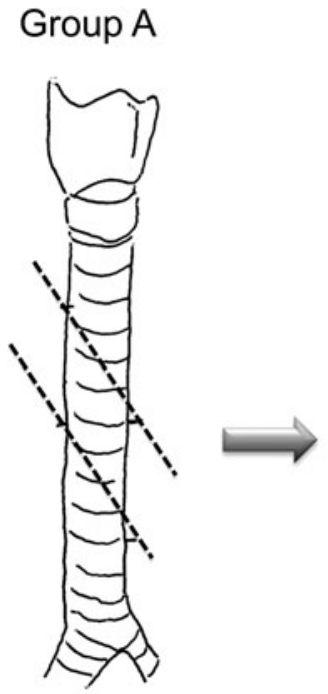

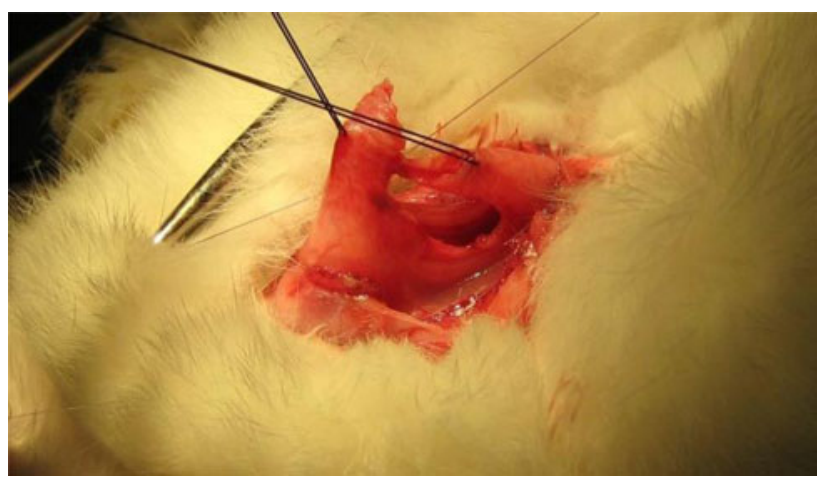

Fig. 2 The slant side-to-side tracheal anastomosis (slanted from the front to the back)

\section{Results}

All the rabbits were still alive until the 10th week after surgery. The animals were classified into three groups: in group A, the front to the back $(n=4)$, in group B, right to left $(n=4)$, and in group C, no surgery was performed and controls $(n=3)$. Within 1-2 weeks after surgery, the rabbits began to wheeze which might have been the result of spontaneous narrowing of the tracheas due to edema but no additional complications were noted.

\section{Body weight}

In group A, animal no. 1 had poor weight gain, but the others gained weight in a manner similar to the control group (the average of 3 rabbits). In group B, animal no. 4 had poor weight gain, however, the others gained body weight in a manner similar to the control group (the average of 3 rabbits). There were no major differences between groups $\mathrm{A}$ and $\mathrm{B}$ with regard to body weight gain (Figs. 3, 4).

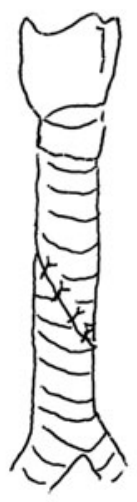

\section{Group B}

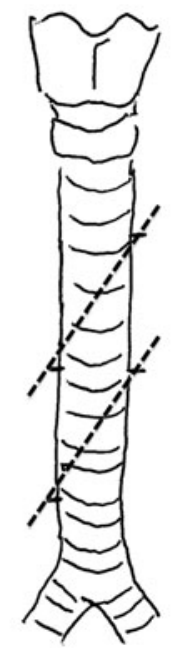

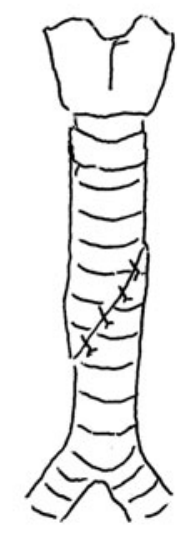




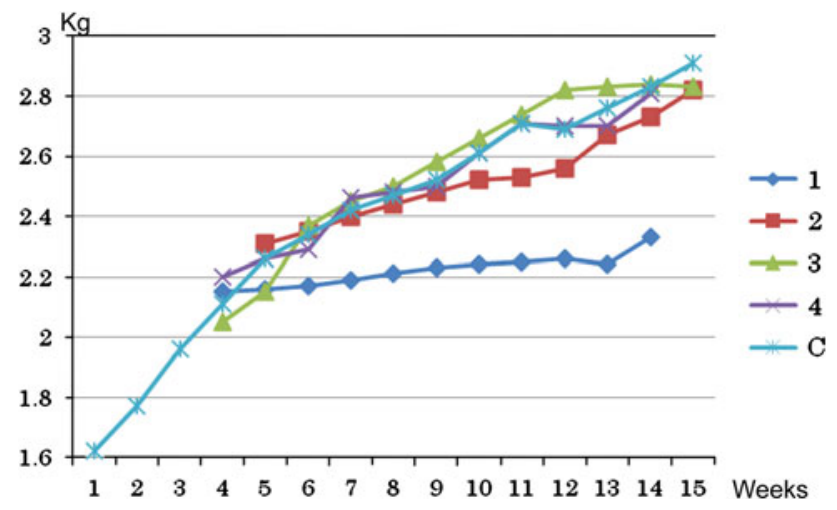

Fig. 3 The body weight gain in group A

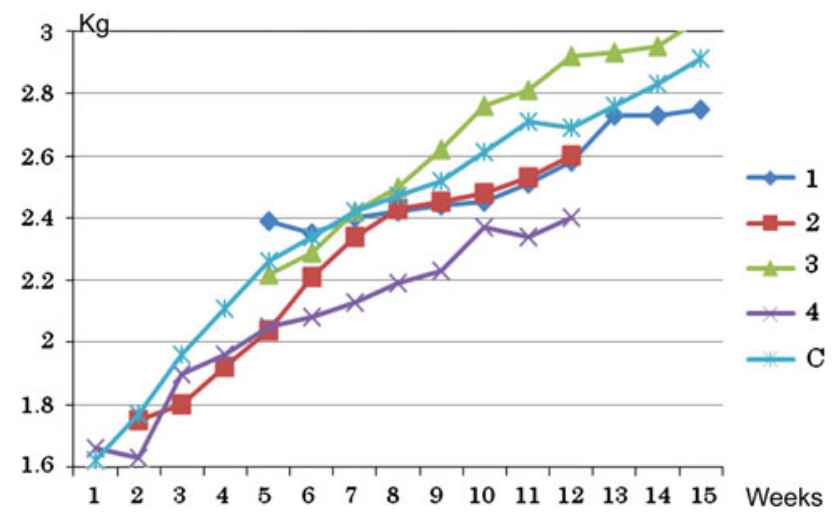

Fig. 4 The body weight gain in group B

Tracheal growth

Table 1 shows the external diameter of the trachea after surgery at 10-11 weeks. The area of the anastomosis was wider than the areas above and below. This was because of the presence of a hyperplastic scar. In group B, the external diameter of the anastomosis was smaller than that in group A. However, we measured the diameter from the right side

Table 1 Changes in the tracheal diameter

\begin{tabular}{clll}
\hline & $\begin{array}{l}\text { Diameter } \\
\text { of upper } \\
\text { anastomosis } \\
(\mathrm{mm})\end{array}$ & $\begin{array}{l}\text { Diameter of } \\
\text { anastomosis } \\
(\mathrm{mm})\end{array}$ & $\begin{array}{l}\text { Diameter } \\
\text { of lower } \\
\text { anastomosis } \\
(\mathrm{mm})\end{array}$ \\
\hline Group A & & 10 & \\
1 & 8 & 10 & 8 \\
2 & 8 & 10 & 8 \\
3 & 7 & 9 & 7 \\
4 & 8 & & 7 \\
Group B & & 9 & 8 \\
1 & 8 & 8 & 8 \\
2 & 7 & 9 & 8 \\
3 & 7 & 9 & 7 \\
4 & 8 & & \\
\hline
\end{tabular}

to the left side, so in group A the estimate was likely larger than the actual diameter.

Bronchoscopy

We were able to identify the suture line from front to back in group A and from right to left in group B. The inner walls of the trachea were smooth, and there were no disconnections or granulation areas (Fig. 5). Even in the two rabbits that exhibited poor weight gain, there were no special findings.

Histology

There was no evidence of differential growth in any part of the circumference of the normal and anastomotic tracheas. In some animals, the cross-section was not completely circular but assumed a more ovoid shape, especially at the level of the side-by-side anastomosis. Histologically, all tracheal lumina were completely lined with normal respiratory epithelium and normal surrounding cartilage (Figs. 6, 7). The suture materials did not lead to any remarkable reactions around the suture area at the time of harvest, 10 weeks after the surgical procedure. We could not identify any histological differences between group A and group B.

\section{Discussion}

Slide tracheoplasty offers several clinical advantages for long congenital tracheal stenosis in small infants [1]. Reconstruction is performed with the patient's native tracheal tissues, so the post-operative problems associated with graft materials are avoided [2, 3]; even very elongated stenosis, such as an $80 \%$ lesion, requires shortening of only half of the length of the stenosis, and this avoids reconstruction under tension [4]; the increase obtained by doubling the circumference appears to be adequate to provide marked and nearly complete symptomatic relief. However, because of its recent introduction into clinical practice and the absence of a long-term follow-up, the effects of growth on the luminal size are not yet known.

We performed slide tracheoplasty on growing rabbits because their tracheas resemble the human tracheas anatomically and functionally, and their growth during the early periods parallel the somatic growth of infants and children. The entire trachea was exposed through a midline cervical incision, and one-third of the estimated length of the trachea was resected in a slanting manner at an appropriate angle. We then performed a slant side-to-side tracheal anastomosis in two different ways using growing rabbits. We consider that this animal model of slide 


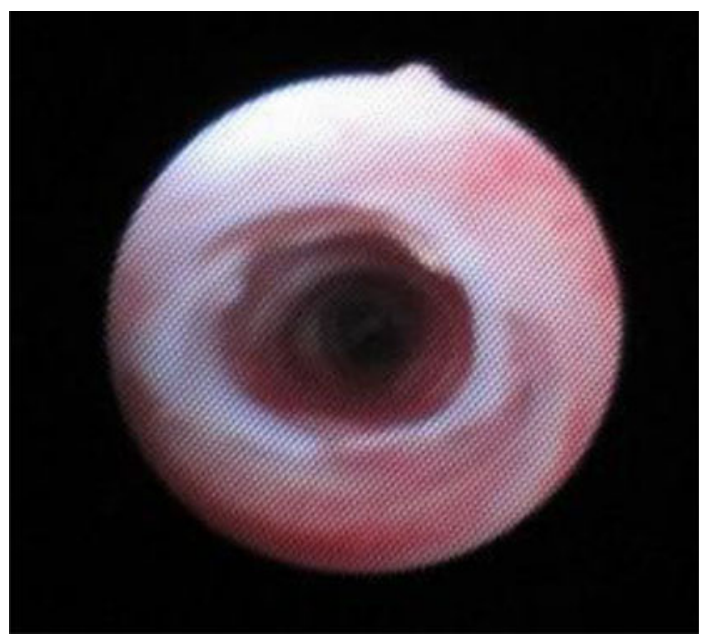

Fig. 5 The bronchoscopic findings of a representative animal (no. 4 in group B). The inner walls of the trachea were smooth, and there were no disconnections or granulation areas
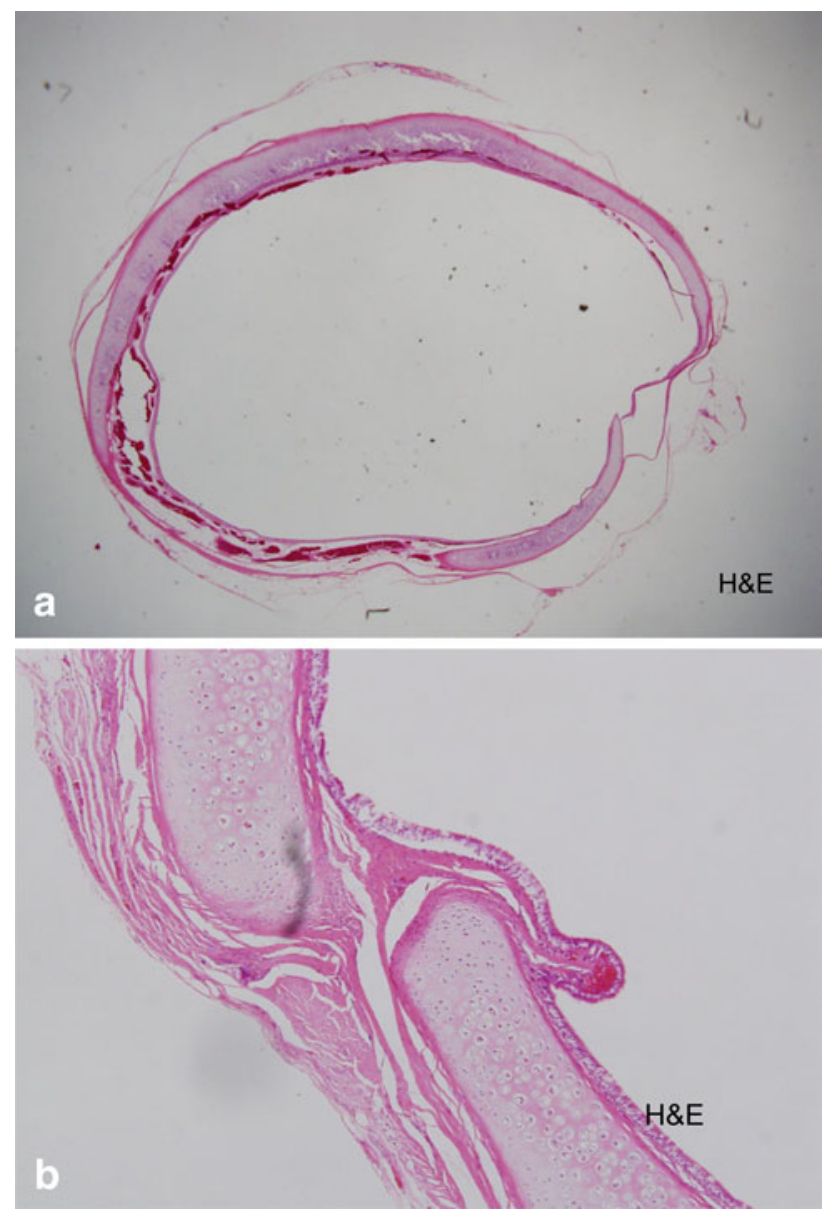

Fig. 6 a Hematoxylin and eosin staining of the reconstructed trachea. The section taken at the midpoint of the suture line revealed no granulation, and efficient patency and continuity of the respiratory epithelium. b The suture materials did not lead to any remarkable reactions around the suture area at the time of harvest, 10 weeks after the surgical procedure

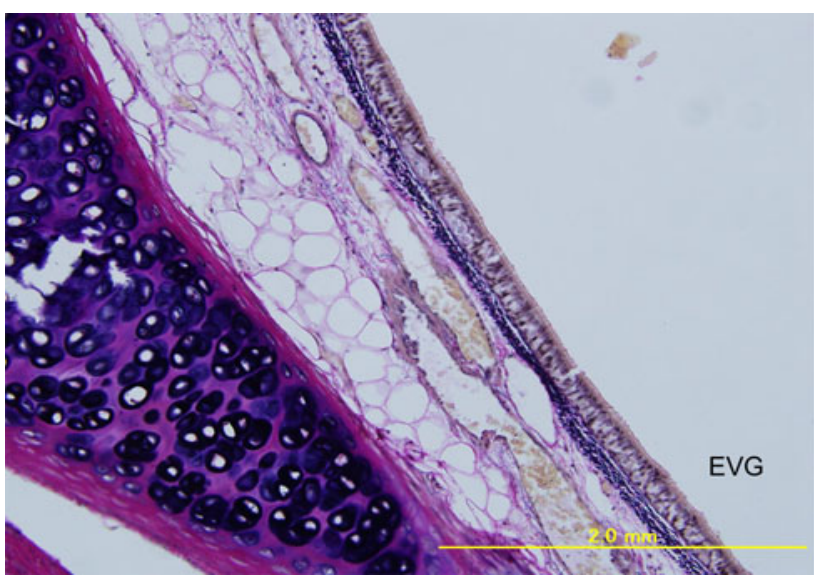

Fig. 7 Elastica van Gieson staining revealed efficient vessels and red blood cells in the vessel

tracheoplasty is very similar to human slide plasty in long congenital tracheal stenosis.

The results presented herein provide evidence that slide tracheoplasty does not impair or distort tracheal growth, even when there was involvement of up to $30 \%$ of the investigated trachea.

The external diameter of trachea was wide; we therefore think that the tension of the anastomoses was low, and the scar was hyperplastic. In group B, the external diameter of the anastomosis was smaller than that in group A. However, we measured the diameter from the right side to the left side, so in group A the estimated diameter was likely to be larger than the true diameter.

By bronchoscopy, we noted that there were no major differences between the surgical site and normal tracheas in either group. Histologically, all tracheas were intact when the animals were euthanized, and their lumina were epithelialized with normal respiratory epithelium even over the reconstructed surfaces. In our study, two animals exhibited poor weight gain (no. 1 in group A and no. 4 in group B). Regarding animal no. 1 in group A, we thought that our surgical skills for performing this surgery might not have been optimal, since this was the first animal treated. For animal no. 4 in group B, this animal was the most lightweight prior to the surgery, so the stress of the operation may have been stronger in this animal. However, these are just the possible explanations, and we do not have any evidence for why these animals had poor weight gain.

The impact of slide tracheoplasty on the tracheal blood supply has not been previously evaluated in human cases. Although Grillo [5] modified the original technique to maintain the blood supply better, Tsang and colleagues [1] noted no ischemic problems after circumferential mobilization. In our study, we were able to microscopically identify sufficient vessels (Fig. 6) and also did not observe any ischemic problems, regardless of the efficiency of 
circumferential mobilization. In our study, because of the length of the reconstructed trachea and to ensure a tensionfree anastomosis, we minimized the tracheal dissection, but were obligated to perform a circumferential mobilization of $>60 \%$ of the investigated trachea.

In conclusion, we performed slant tracheal anastomosis in two different ways on growing rabbits as an animal model of slide tracheoplasty. The results indicated that these two methods did not prevent the tracheal growth, and the trachea did not buckle macroscopically. Neither type of slide tracheoplasty interrupted the growth of the trachea. Based on these results, it can be concluded that slide tracheoplasty should be considered as an efficient tracheoplasty technique for neonates or infants with long congenital tracheal stenosis.

Open Access This article is distributed under the terms of the Creative Commons Attribution Noncommercial License which permits any noncommercial use, distribution, and reproduction in any medium, provided the original author(s) and source are credited.

\section{References}

1. Tsang V, Murday A, Gillbe C, Goldstraw P (1989) Slide tracheoplasty for congenital funnel tracheal stenosis. Ann Thorac Surg 48:623-635

2. Tsugawa C, Nishijima E, Muraji T, Satoh S, Takamizawa S, Yamaguchi M, Yoshimura N, Oka S, Kimura K (2003) Tracheoplasty for long segment congenital tracheal stenosis: analysis of 29 patients over two decades. J Pediatr Surg 38:1703-1706

3. Abdelkafy M, Atriby E, Iskandar M, Mattox E, Mansour A (2007) Slide tracheoplasty applied to acquired subglottic and upper tracheal stenosis. Arch Otolaryngol Head Neck Surg 133:327-330

4. Macchiarini P, Dulmet E, Montpreville V, Mazmanian G, Chapelier A, Dartevelle P (1997) Tracheal growth after slide tracheoplasty. J Thorac Cardiovasc Surg 113:558-566

5. Grillo C (1994) Slide tracheoplasty for long-segment congenital tracheal stenosis. Ann Thorac Surg 58:613-621 